\title{
Características clínicas y patológicas de pacientes con tumores del sistema nervioso central en un centro en Suramérica desde 2010 hasta 2015
}

Clinico-pathological characteristics of central nervous system tumors in a South American center from 2010 to 2015

Esteban Jaramillo-Jiménez ${ }^{1}$ ORCID, Cristian Vargas-García ${ }^{2}$ RCID, lader Rodríguez-Márquez ${ }^{3}$ ORCID, Juliana Sandoval-Barrios4, David Gómez-Duque ${ }^{5}$, Hernán Barrientos-Montoya ${ }^{6}$, Julián ArangoVélez7, Tatiana Gómez-Escobar, Mónica Massaro-Ceballos9 ORCID, Marta Jiménez-Jaramillo ${ }^{10}$

Fecha correspondencia: Recibido: febrero 20 de 2019. Revisado: junio 5 de 2020. Aceptado: junio 23 de 2020.

Forma de citar: Jaramillo-Jiménez E, VargasGarcía C, Rodríguez-Márquez I, Sandoval-Barrios J, GómezDuque D, Barrientos-Montoya H, et al. Características clínicas y patológicas de pacientes con tumores del sistema nervioso central en un centro en Suramérica desde 2010 hasta 2015. Rev CES Med. 2020; 34(2): 103-113.

\section{Open access}

(C) Derecho de autor

Licencia creative commons

Ética de publicaciones

Revisión por pares

Gestión por Open Journal System DOl: http://dx.doi.org/10.21615/ cesmedicina.34.2.2

Comparte

AOG.

\section{Resumen}

Objetivo: caracterizar los pacientes diagnosticados con tumores del sistema nervioso central en el Instituto Neurológico de Colombia durante el periodo 2010-2015. Métodos: estudio descriptivo retrospectivo. Para los tumores primarios se usó la clasificación de la Organización Mundial de la Salud y para los metastásicos se usó la Clasificación Internacional de Enfermedades en Oncología. Resultados: se identificaron 288 pacientes, 194 de ellos con tumores primarios y 94 tumores metastásicos. No se lograron clasificar los tumores primarios en el $23,7 \%$ de los casos y para los metastásicos no se obtuvo la clasificación morfológica (histológica) en el $35,1 \%$ de los casos. Los hombres presentaron con mayor frecuencia tumores de comportamiento maligno tipo glioblastoma NEO (no especificado de otra manera) (14,9\%) y en las mujeres predominaron los tumores de comportamiento benigno tipo meningioma (23,2 \%). En mayores de 65 años, tanto el glioblastoma NEO como el meningioma fueron más frecuentes en mujeres con $17,4 \%$ y $28,3 \%$, respectivamente. Entre los pacientes con tumores metastásicos, de acuerdo con la clasificación topográfica, los tumores primarios más frecuentes se localizaron en pulmón $(39,4 \%)$ y mama (17\%). No se identificó el sitio primario de metástasis en el 11,7\% de los casos. La histología más comúnmente identificada fue el adenocarcinoma (14,9\%), seguido del carcinoma (8,5\%). Conclusiones: para una vigilancia efectiva de la enfermedad es necesario realizar un monitoreo epidemiológico y clínico de tumores primarios y metastásicos, mediante el uso de registros institucionales de cáncer, incluyendo datos topográficos, histológicos y moleculares, según disponibilidad.

Palabras clave: Neoplasias del sistema nervioso; Neoplasias encefálicas; Epidemiología; Patología.

\section{Abstract}

Objective: The aim of this study was to characterize patients with a diagnosis of a central nervous system (CNS) tumors at the Instituto Neurologico de Colombia during the period between 2010 to 2015. Methods: A retrospective 
ISSN 0120-8705

e-ISSN 2215-9177

\section{Sobre los autores:}

1. MD, Neurología oncológica. Unidad de Neuro-Oncología, Instituto Neurológico de Colombia (INDEC) I Universidad CES. Grupo de investigación GrupLAC Colciencias INDEC-CES. Medellín-Colombia.

2. MD, MSc en Epidemiología e investigación clínica. Investigación en atención primaria en salud, Facultad de Medicina, Universidad de Notre Dame, Australia.

\section{MD, MSc en}

Epidemiología. Centro de Investigación, Instituto Neurológico de Colombia (INDEC). Grupo de investigación GrupLAC Colciencias INDEC-CES. Medellín-Colombia.

4. MD, Unidad de Neuro-
Oncología, Instituto
Neurológico de Colombia
(INDEC). Medellín-Colombia.

5. MD, Radioterapia Radiocirugía, Unidad de Radiocirugía Robótica CyberKnife, Instituto Neurológico de Colombia (INDEC). Medellín-Colombia.
6. MD, Neurocirugía - Radiocirugía, Unidad de Radiocirugía Robótica CyberKnife, Instituto Neurológico de Colombia (INDEC). Medellín-Colombia.

descriptive study was conducted. The Classification of the World Health Organization was used for CNS primary tumors whereas the International Classification of Diseases for oncology (ICD-0) was used for CNS metastatic tumors. Results: 288 patients were identified, 194 of them with primary tumors of CNS and 94 with metastatic tumors from systemic cancer. It was not possible to classify primary tumors in 23.7 $\%$ of the cases and regarding the metastatic tumors it was not possible to obtain the classification in $35.1 \%$ of the cases. Men presented more frequently tumors of malignant behavior such as glioblastoma NOS (not otherwise specified) (14.9\%) while in women benign behavior tumors such as meningioma predominated (23.2\%). For population older than 65 years old, both glioblastoma NOS and meningioma were more frequent in women with $17.4 \%$ and $28.3 \%$, respectively. In patients with CNS metastatic tumors, according to the topographic classification, the most frequent primary tumors were lung (39.4\%), followed by breast (17\%). Its origin was not identified in $11.7 \%$ of the cases. The most identified histology was adenocarcinoma (14.9\%), followed by carcinoma (8.5 \%). Conclusion: For disease surveillance, it is necessary to complete epidemiological and clinical monitoring of primary and metastatic tumors of the CNS by using institutional cancer registries including topographic, histological and molecular data according to availability.

Keywords: Nervous System Neoplasms; Brain Neoplasms; Epidemiology; Pathology.

\section{Introducción}

La incidencia global de tumores primarios del sistema nervioso central es de 10,8 por 100000 personas-año y la tasa de tumores malignos oscila entre 5,71 y 5,78 casos por 100000 personas-año, siendo mayor en la población adulta. Se ha encontrado gran variabilidad por regiones, con un mayor número de casos en Europa. En América del Sur se ha notado un incremento en el número de casos, específicamente en Ecuador, Brasil y Colombia con incrementos anuales hasta del 3,3 \%, 1,3 \% y 1,1\%, respectivamente (1-3).

Las metástasis son los tumores más frecuentes (tanto supra como infratentoriales) del sistema nervioso central (4) y originados con mayor frecuencia en pulmón (39 \% - $56 \%)$, mama (13\% -19\%) y por melanoma (6 \% - $11 \%)$. Las metástasis cerebrales son diagnosticadas, cada vez con mayor frecuencia, en personas asintomáticas como resultado de un estudio de tamización. En los casos donde se presentan síntomas neurológicos, estos dependerán de la localización, tamaño y edema del tumor. El pronóstico de estos pacientes es ominoso, con una sobrevida de 1-2 meses sin ningún tipo de intervención. Sin embargo, dentro de los factores pronósticos que condicionan la sobrevida se deben tener en cuenta el estado funcional, la edad, metástasis extracraneales; número y volumen de la metástasis cerebrales y el control del tumor primario $(5,6)$.

En Colombia, la mortalidad por tumores malignos del sistema nervioso central se ha ido incrementando, a diferencia de lo ocurrido en otros países (7). Las tasas de incidencia son probablemente subestimadas, especialmente según el subtipo histológico, además de la variabilidad en las líneas celulares de origen (8).

La Organización Mundial de la Salud (OMS) y su Agencia Internacional de Investigación del Cáncer (por sus siglas en inglés: IARC) recomiendan para estimar la incidencia y distribución de los tumores en una población determinada, el uso de registros poblacionales de cáncer, en los cuales se registra la información referente a nuevos casos dentro de un área geográfica determinada y en un período de tiempo específico. Otra 
7. MSc en Telesalud. Gestión de Información, Clínica Universitaria Bolivariana I Universidad Pontificia Bolivariana (UPB). MedellínColombia.

\section{MD, Residente de Neurología Clínica, Universidad CES, Medellín, Colombia.}

\section{MD, MSc en} Epidemiología. Investigación e Innovación Medicina, Universidad CES.

10. MD, Neurofisiología, Dirección General, Instituto Neurológico de Colombia (INDEC). MedellínColombia.

En Colombia, la mortalidad por tumores malignos del sistema nervioso central se ha ido incrementando, a diferencia de lo ocurrido en otros países. herramienta útil para la estandarización de la enfermedad ha sido la implementación de la Clasificación Internacional de Enfermedades para Oncológica (CIE-O) que permite la codificación de la localización y la histología de la neoplasia. Desde el año 2016, la OMS adicionó parámetros moleculares para clasificar estos tumores y surge un nuevo subgrupo denominado "NEO" (no especificado de otra manera), para aquellas regiones geográficas sin capacidad para completar análisis moleculares $(9,10)$.

Teniendo en cuenta la complejidad de los tumores del sistema nervioso central, los desafíos para una adecuada clasificación histo-molecular y la carencia de información epidemiológica certera a nivel local, es importante conocer dicho perfil en instituciones especializadas para optimizar los estándares de vigilancia y orientar la toma de decisiones clínicas y administrativas. El objetivo del presente estudio fue caracterizar por clínica e histopatología los pacientes con tumores del sistema nervioso central tratados en la institución.

\section{Materiales y métodos}

Se realizó un estudio descriptivo retrospectivo en pacientes atendidos por lesiones tumorales del sistema nervioso central en el Instituto Neurológico de Colombia en Medellín, desde el año 2010 hasta el 2015. Se empleó un muestreo por conveniencia de la población atendida durante dicho periodo con al menos uno de los siguientes códigos de la Clasificación Internacional de Enfermedades (CIE-10) (11): C47, C70, C71, C72, C751, C752, C753, C793, C794, D166, D32, D33, D352, D353, D354, D361, D42, D43, D443, D444, D445, G130, G131 y G731.

Se incluyeron todos los tumores primarios (no malignos y malignos) y metastásicos diagnosticados entre 2010 y 2015, ubicados en cerebro, meninges, médula espinal, pares craneales, otras partes del sistema nervioso central, glándula pituitaria, glándula pineal y tumores olfativos de la cavidad nasal. Se excluyeron los registros de aquellos pacientes con información insuficiente para la clasificación de la lesión tumoral.

Para la recolección de la información se hizo una revisión exhaustiva de los registros de la historia clínica institucional. Posteriormente, se realizó una validación mediante el uso del Registro Poblacional de Cáncer de Antioquia, complementando la información histológica para cada caso según disponibilidad. Los tumores primarios fueron validados según la clasificación de tumores del sistema nervioso central de la OMS 2016 (10) y las metástasis fueron clasificadas según la CIE-O versión 3 (9). Los datos obtenidos se combinaron en un solo conjunto. Se eliminaron registros del conjunto de datos finales por uno o más de los siguientes motivos: combinación de sitio / histología no válida o no realizada, registros duplicados y errores en la secuencia temporal del diagnóstico. Para los análisis estadísticos se utilizó STATA $14{ }^{\circledR}$ (StataCorp, College Station, TX, USA).

Se realizó un análisis descriptivo para tumores primarios y metastásicos. El estado funcional de los pacientes fue evaluado en el momento de ingreso a la institución de acuerdo al índice de Karnofsky (12) para los tumores primarios y con la escala ECOG (siglas de Eastern Cooperative Oncology Group) (13) para los tumores metastásicos.

Se presentan medidas de tendencia central de acuerdo con la naturaleza de la variable. Se determinó la normalidad de la variable edad mediante el test Shapiro-Francia y se describió la media con su respectiva medida de dispersión, luego, fue recategorizada en menores de 18 años, 18 a 65 años y mayores de 65 años. Los resultados se presentaron por subgrupos de edad y sexo. 
Los hombres presentaron con mayor frecuencia tumores de comportamiento maligno tipo glioblastoma NEO, así como otros astrocitomas; mientras que en las mujeres predominaron los tumores de comportamiento benigno tipo meningioma.
Este estudio fue aprobado por el Comité de Ética en investigación clínica en seres humanos del Instituto Neurológico de Colombia.

\section{Resultados}

Inicialmente, se identificaron 254 pacientes con tumores primarios y 94 con metástasis al sistema nervioso central con base en la clasificación CIE-10. Posteriormente, el $76,3 \%$ de los casos con tumores primarios fueron validados con base en la clasificación de tumores del sistema nervioso central de la OMS 2016 y todos los casos de lesiones metastásicas fueron clasificadas según la CIE-0 versión 3.

\section{Tumores primarios del sistema nervioso central}

De los 194 casos de tumores primarios e identificados y validados, el 51,6\% se presentaron en mujeres. La edad promedio al diagnóstico fue de $51 \pm 17$ años, encontrándose un 2,6\% de los casos en menores de 18 años, 73,7\% entre los 18 y 65 años y $23,7 \%$ en mayores de 65 años. El $56,2 \%$ de los pacientes pertenecían al régimen de salud contributivo y $36,6 \%$. al subsidiado. El 35,1 \% de los pacientes ingresó por consulta externa, 34,5 \% fueron remitidos desde otras instituciones y 30,4 \% ingresó por urgencias.

El $16 \%$ de la población estudiada obtuvo una puntuación en la escala de funcionalidad de Karnosky $\geq 70 \%$, presentando focalización neurológica susceptible de rehabilitación en el 46,9 \% y epilepsia tumoral como debut clínico en el 30,4 \% de los casos. El estudio de neuroimagen ordenado inicialmente correspondió a la tomografía computarizada en el $61,9 \%$ de los casos y al 8,8 \% de los pacientes se les ordenó neuroimagen avanzada. A un tercio de los pacientes se les ordenó inmunohistoquímica.

Al 81,4 \% de los pacientes se les realizó intervención quirúrgica, de los cuales a 52,9 \% se les practicó resección total, 19,7 \% resección parcial y 9,3 \% fueron llevados a biopsia. El 5,2 \% de los casos presentaron complicaciones posquirúrgicas inmediatas. Se realizó radioterapia en $18,7 \%$ de los pacientes y quimioterapia al 9,3 \%.

Los hombres presentaron con mayor frecuencia tumores de comportamiento maligno tipo glioblastoma NEO, así como otros astrocitomas; mientras que en las mujeres predominaron los tumores de comportamiento benigno tipo meningioma. Por otro lado, el adenoma de hipófisis presentó distribución similar en ambos sexos (figura 1).

En el cuadro 1 se presentan los datos histológicos de pacientes con tumores primarios del sistema nervioso central en relación con grupos etarios. La distribución de los meningiomas durante el periodo de estudio se presenta en el cuadro 2. 


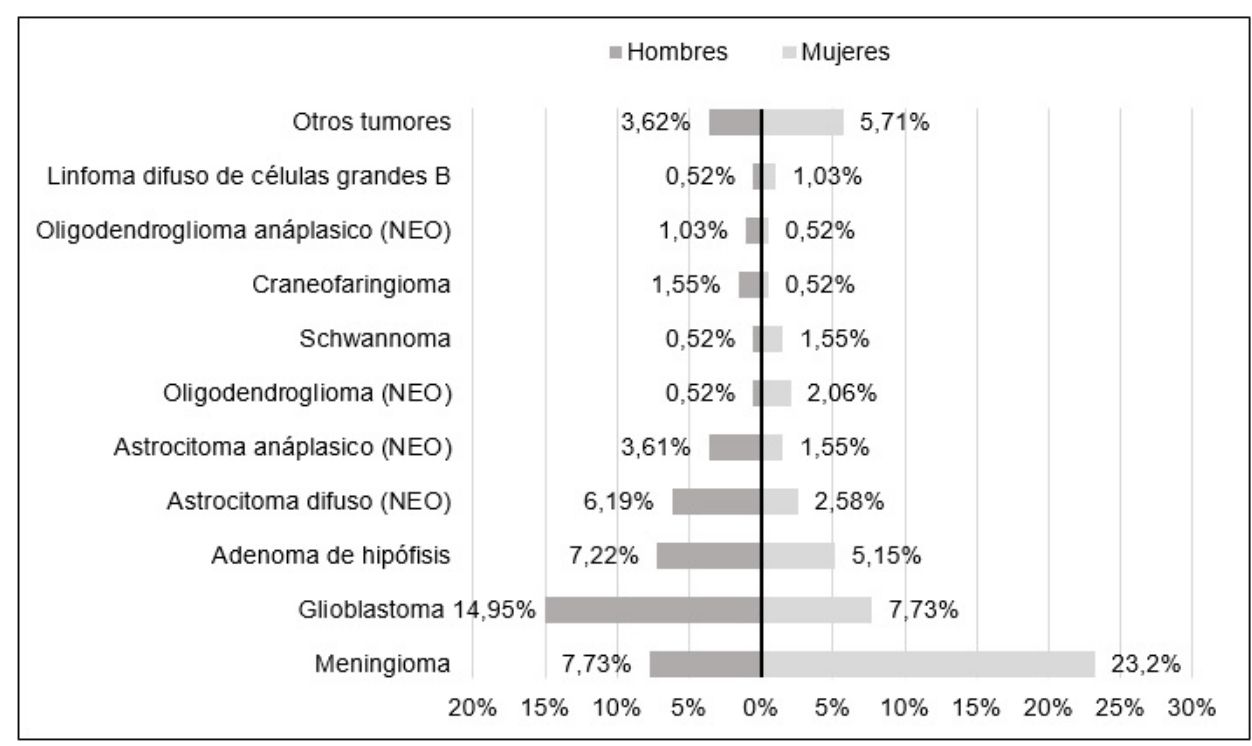

Figura 1. Histología de pacientes con tumores primarios del sistema nervioso central según sexo, Instituto Neurológico de Colombia, 2010-2015 ( $n=194)$

Cuadro 1. Histología por grupo etario de pacientes adultos con tumores primarios del sistema nervioso central según sexo, Instituto Neurológico de Colombia, 2010-2015

\begin{tabular}{|c|c|c|c|c|c|c|}
\hline \multirow{3}{*}{ Histología } & \multicolumn{3}{|c|}{$18-65$ años } & \multicolumn{3}{|c|}{ Mayores de 65 años } \\
\hline & Total & Hombres & Mujeres & Total & Hombres & Mujeres \\
\hline & $\begin{array}{c}100 \% \\
(n=143)\end{array}$ & $\begin{array}{l}49,6 \% \\
(n=71)\end{array}$ & $\begin{array}{l}50,2 \% \\
(n=72)\end{array}$ & $\begin{array}{c}100 \% \\
(n=46)\end{array}$ & $\begin{array}{l}43,5 \% \\
(n=20)\end{array}$ & $\begin{array}{l}56,5 \% \\
(n=26)\end{array}$ \\
\hline Meningioma & 28,7 & 6,3 & 22,4 & 41,3 & 13,0 & 28,3 \\
\hline Glioblastoma (NEO) & 18,9 & 14,0 & 4,9 & 36,9 & 19,6 & 17,3 \\
\hline Adenoma de hipófisis & 11,9 & 7,7 & 4,2 & 15,2 & 6,5 & 8,7 \\
\hline Astrocitoma difuso (NEO) & 11,2 & 7,7 & 3,5 & - & - & - \\
\hline Astrocitoma anaplásico (NEO) & 6,3 & 4,2 & 2,1 & - & - & - \\
\hline Oligodendroglioma (NEO) & 3,5 & 0,7 & 2,8 & - & - & - \\
\hline Schwannoma & 2,8 & 0,7 & 2,1 & - & - & - \\
\hline Craneofaringioma & 2,8 & 2,1 & 0,7 & - & - & - \\
\hline $\begin{array}{l}\text { Oligodendroglioma } \\
\text { anaplásico (NEO) }\end{array}$ & 1,4 & 0,7 & 0,7 & 2,2 & 2,2 & 0,0 \\
\hline Oligoastrocitoma anaplásico & 1,4 & 0,7 & 0,7 & - & - & - \\
\hline Astrocitoma pilocítico & 1,4 & 0,7 & 0,7 & - & - & - \\
\hline Hemangioblastoma & 1,4 & 0,7 & 0,7 & - & - & - \\
\hline Otros tumores & 8,3 & 3,5 & 4,8 & 4,4 & 2,2 & 2,2 \\
\hline
\end{tabular}


Cuadro 2. Pacientes con meningiomas diagnosticados en el Instituto Neurológico de Colombia, 2010-2015 ( $n=60)$

\begin{tabular}{lc}
\hline \multicolumn{1}{c}{ Meningiomas } & $\%$ \\
\hline Sin subtipo histológico identificado & 36,6 \\
\hline Meningotelial & 25,0 \\
\hline Transicional & 15,0 \\
\hline Atípico & 10,0 \\
\hline Angiomatoso & 8,3 \\
\hline Fibroso & 1,7 \\
\hline Psamomatoso & 1,7 \\
\hline De células claras & 1,7 \\
\hline
\end{tabular}

Entre los 94 pacientes diagnosticados con tumores metastásicos al sistema nervioso central, el 61,7\% eran mujeres; la edad promedio fue de $60 \pm 14$ años. Los tumores primarios más frecuentes fueron en pulmón, mama, piel y riñón.

\section{Tumores secundarios del sistema nervioso central}

Entre los 94 pacientes diagnosticados con tumores metastásicos al sistema nervioso central, el 61,7 \% eran mujeres. La edad promedio fue de $60 \pm 14$ años, siendo el grupo entre los 18 y 65 años el de mayor proporción (60,6 \%) y no se encontraron menores de edad con tumores metastásicos. El tipo de afiliación más común fue el contributivo (45,7\%), seguido del subsidiado (25,5\%). En cuanto al servicio de admisión, los más frecuentes fueron la consulta externa $(45,7 \%$ ) y urgencias $(30,9 \%)$.

Los pacientes con tumores metastásicos presentaron síntomas de focalización neurológica en el $56,4 \%$ de los casos y $18,1 \%$ debutaron con crisis convulsivas. Se clasificaron 73 pacientes según la escala ECOG, y de estos, el 78,1 \% tenían un puntaje mayor o igual a uno. Los estudios imagenológicos ordenados inicialmente fueron la resonancia magnética (50 \%) y seguido de la tomografía (47,9\%). Realizaron neuroimágenes avanzadas en el 9,6\%.

El 35,1 \% de los pacientes con metástasis cerebrales fueron sometidos a intervención quirúrgica; de estos en el 18,1 \% se practicó resección total, en el 8,5 \% resección parcial y $6,4 \%$ fueron llevados a biopsia. Se presentaron dos casos de hematomas como complicaciones postquirúrgicas inmediatas. El 52,1 \% de los pacientes con metástasis cerebrales fueron tratados con radioterapia, la técnica más utilizada fue la radiocirugía $(45,7 \%)$. De igual forma, el $50 \%$ de los pacientes recibieron quimioterapia sistémica.

Al realizar la clasificación topográfica se encontró que los tumores primarios más frecuentes fueron en pulmón (39,4\%), mama (17\%), piel $(9,6 \%)$ y riñón (5,3 \%). No se logró identificar el tumor primario en el 11,7 \% de los casos. Se observó una mayor proporción de tumores primarios de pulmón en mujeres (23,4 vs. $16 \%$ ). Mientras que el tumor primario en piel fue más frecuente en hombres $(6,4 \%)$ que en mujeres $(3,2 \%)$ (figura 2$)$.

En cuanto a la clasificación histológica no se obtuvieron datos concluyentes en el $35,1 \%$ de los pacientes con tumores metastásicos. La histología más común fue el adenocarcinoma (14,9\%), seguido de carcinoma (8,5\%), carcinoma ductal infiltrante (7,5 \%) y melanoma maligno (5,3 \%). En cuanto a la distribución según sexo, el adenocarcinoma fue más frecuente en hombres, mientras que para las mujeres fue el carcinoma (figura 3). 


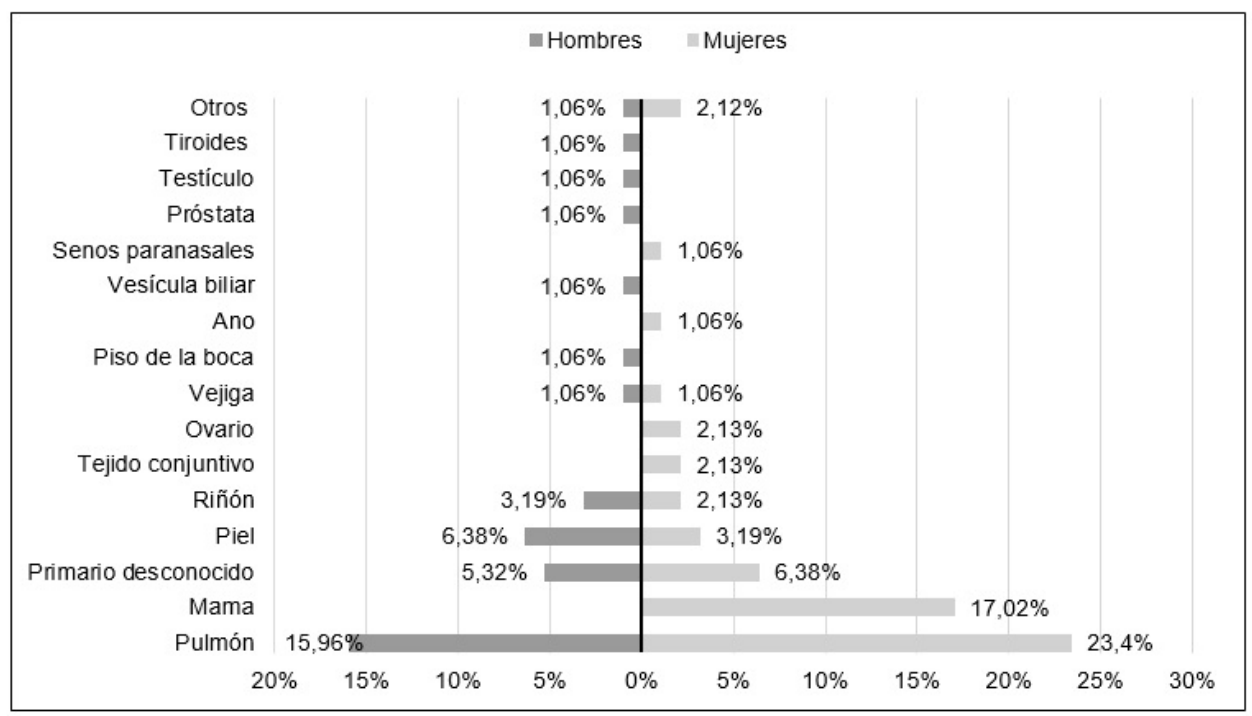

Figura 2. Topografía del tumor primario en pacientes con tumores metastásicos al sistema nervioso central según sexo, Instituto Neurológico de Colombia, 2010$2015(n=94)$

Se han logrado identificar dos picos de incidencia para tumores primarios del sistema nervioso central relacionados con la edad, el primero correspondiente a menores de cinco años y el segundo luego de los 55 años.

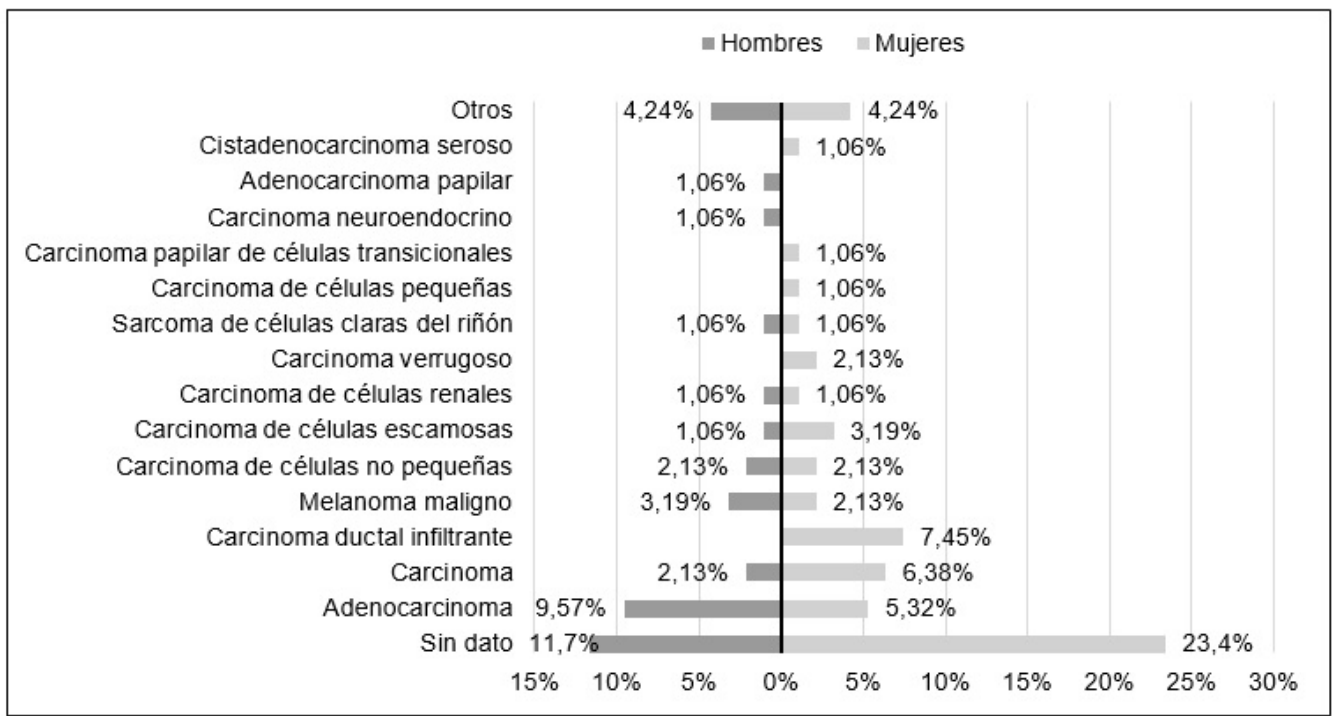

Figura 3. Histología del tumor en pacientes con tumores metastásicos al sistema nervioso central según sexo, Instituto Neurológico de Colombia, 2010-2015 (n=94)

\section{Discusión}

Este estudio realizó un monitoreo epidemiológico y clínico de tumores primarios y metastásicos al sistema nervioso central con la finalidad de construir una estadística institucional que oriente las conductas clínicas y administrativas. Con los datos disponibles se lograron establecer algunos hallazgos de cara al propósito planteado.

Se encontró que los tumores primarios del sistema nervioso central fueron diagnosticados con mayor frecuencia en la población adulta femenina, algo que resulta coincidente con lo hallado en otras investigaciones, con proporciones que oscilan entre 53,3 \% a 58,5 \% (14-16). No obstante, es necesario señalar la existencia de 
Los hombres presentaron una mayor frecuencia de tumores primarios del sistema nervioso central de comportamiento maligno tipo glioblastoma NEO. En las mujeres predominaron los tumores de comportamiento benigno tipo meningioma. resultados contrarios a los expuestos, donde se reportan tasas ajustadas por edad para Colombia de 3,4 casos por 100000 habitantes en hombres; mientras que en mujeres correspondió 2,5 casos por 100000 habitantes (17). Algo que podría explicar estos hallazgos contradictorios sería el tipo de fuentes de información empleada, el perfil de la población atendida, el análisis de solo tumores malignos de sistema nervioso central y la variabilidad geográfica de la enfermedad.

Se han logrado identificar dos picos de incidencia para tumores primarios del sistema nervioso central relacionados con la edad, el primero correspondiente a menores de cinco años y el segundo luego de los 55 años $(18,19)$. Nuestros hallazgos revelan una mayor proporción en la población adulta. Lo encontrado podría ser explicado por factores epigenéticos propios de esta región del país, así como por el tipo de población atendida en la institución.

En este estudio, los hombres presentaron una mayor frecuencia de tumores primarios del sistema nervioso central de comportamiento maligno tipo glioblastoma NEO. En las mujeres predominaron los tumores de comportamiento benigno tipo meningioma. Algunos estudios apoyan tales resultados (16,20). Los meningiomas aumentan su presentación con el incremento de la edad, observándose un pico entre los 40 y 85 años. Posteriormente, la frecuencia de los meningiomas tiende a disminuir (21). En cambio, los glioblastomas presentan una mayor incidencia entre los 60 y 74 años (14), lo que es concordante con nuestros hallazgos. Existen teorías que sugieren la influencia hormonal en el desarrollo de meningiomas (22); según Qi et al. (23) el riesgo de tener meningioma aumenta un $19 \%$ en mujeres que usan terapia de reemplazo hormonal.

En relación con los tumores metastásicos al sistema nervioso central, una cohorte de pacientes con enfermedad oligo-metastásica a cerebro y buen estado funcional informó una proporción en mujeres del 59,6 \% (24). De igual manera, otro estudio realizado en cuatro centros oncológicos reporta una mayor proporción de mujeres con metástasis cerebrales (54 \%) (25). Estos hallazgos son similares a lo reportado en este estudio. Así mismo, algunos estudios han reportado una edad promedio al momento del diagnóstico de metástasis cerebrales de alrededor de 60 años $(24,26)$.

En relación a la clasificación topográfica de los tumores metastásicos al sistema nervioso central, un estudio realizado en Londres reporta que en el 7,8 \% de los casos no se logró identificar el tumor primario (24) y otro estudio menciona que pudiera ascender hasta el 10 \% (15). Se ha reportado como ubicación más frecuente del tumor primario los pulmones, seguido de mama y tracto gastrointestinal $(15,27)$. Dichos hallazgos podrían ser dependientes de la disponibilidad de métodos diagnósticos como mamografía, endoscopia de vías digestivas, tomografía de emisión de positrones, entre otros. Lo anterior, expone la necesidad de enfatizar la búsqueda del tumor primario.

Algunos estudios reportan que el cáncer de mama es la fuente más común de metástasis cerebral en mujeres (5,28); no obstante, en este trabajo se encontró una mayor frecuencia de origen pulmonar. Lo anterior podría relacionarse con el consumo de tabaco, sugiriendo la necesidad de un programa de cesación de tabaco en este grupo poblacional. 
Mayo - agosto de 2020 - Pág 111

Este estudio plantea la necesidad de continuar el monitoreo epidemiológico y clínico de tumores primarios y metastásicos al sistema nervioso central por medio de un registro institucional de cáncer para una adecuada clasificación sistemática, incluyendo análisis moleculares.
Como limitación es necesario señalar que el 23,7 \% de los tumores primarios del sistema nervioso central identificados preliminarmente, no fue posible la clasificación según OMS 2016 y para los tumores metastásicos no se logró la clasificación morfológica según CIE-0 en el 35 \% de los casos. Estas situaciones se explican por la forma cómo se obtuvieron los registros, ya que dicho proceso estuvo basado en la revisión de las historias clínicas que en algunos casos no contenían la información concerniente a las biopsias. En aquellos pacientes con tumores metastásicos la clasificación patológica se obtuvo del tumor primario o de la lesión metastásica. En el primer caso, estos exámenes patológicos se realizaron en otras instituciones, generando dificultades para la consecución de la información. Esta es una situación recurrente en algunas de las investigaciones revisadas y que reportan porcentajes de no identificación del tipo de tumor, que fluctúan entre el $10 \%$ y el $19 \%(15,27,29)$. Se destaca el proceso de validación realizado con el uso del Registro Poblacional de Cáncer de Antioquia.

En conclusión, este estudio plantea la necesidad de continuar el monitoreo epidemiológico y clínico de tumores primarios y metastásicos al sistema nervioso central por medio de un registro institucional de cáncer para una adecuada clasificación sistemática, incluyendo análisis moleculares según disponibilidad, con el fin de aumentar la confiabilidad y utilidad en la toma de decisiones. De esta manera será posible hacer un seguimiento efectivo de la enfermedad con hallazgos relevantes para próximas investigaciones.

\section{Agradecimientos}

Al personal del Registro Poblacional de Cáncer de Antioquia por el soporte en la validación de los datos.

\section{Financiación}

Este trabajo fue financiado por la Dirección de Investigación e Innovación de la Universidad CES bajo la modalidad de mínima cuantía. Este proyecto hace parte del grupo de investigación de Colciencias INDEC-CES con código 125303.

\section{Conflictos de interés}

Ninguno reportado por los autores. Este trabajo fue presentado como ponencia oral en la "Reunión anual de la Sociedad Española de Neurología" en Valencia, España, del 21 al 25 de noviembre de 2017.

\section{Bibliografía}

1. Leece R, Xu J, Ostrom QT, Chen Y, Kruchko C, Barnholtz-Sloan JS. Global incidence of malignant brain and other central nervous system tumors by histology, 2003-2007. Neuro Oncol. 2017;19(11):1553-64.

2. De Robles P, Fiest KM, Frolkis AD, Pringsheim T, Atta C, St. Germaine-Smith C, et al. The worldwide incidence and prevalence of primary brain tumors: a systematic review and meta-analysis. Neuro Oncol. 2015;17(6):776-83.

3. Miranda-Filho A, Piñeros M, Soerjomataram I, Deltour I, Bray F. Cancers of the brain and CNS: global patterns and trends in incidence. Neuro Oncol. 2017;19(2):270-80.

4. Alegría-Loyola MA, Galnares-Olalde JA, Mercado M. Tumores del sistema nervioso central. Rev Med Inst Mex Seguro Soc. 2017;55(3):330-40. 
5. Stelzer K. Epidemiology and prognosis of brain metastases. Surg Neurol Int. 2013;4(5):192-202.

6. Marín A, Renner A, Itriago L, Álvarez M. Metástasis cerebrales: una mirada biológica y clínica. Rev Médica Clínica Las Condes. 2017;28(3):437-49.

7. Piñeros M, Gamboa O, Hernández-Suárez G, Pardo C, Bray F. Patterns and trends in cancer mortality in Colombia 1984-2008. Cancer Epidemiol. 2013;37(3):233-9.

8. Contreras LE. Epidemología de tumores cerebrales. Rev Médica Clínica Las Condes. 2017;28(3):332-8.

9. Fritz A, Shanmugaratnam K. ClE-O: Clasificación Internacional de Enfermedades para Oncología. Pan American Health Org; 2003. 1-242 p.

10. Louis DN, Perry A, Reifenberger G, von Deimling A, Figarella-Branger D, Cavenee WK, et al. The 2016 World Health Organization Classification of Tumors of the Central Nervous System: a summary. Acta Neuropathol. 2016;131(6):803-20.

11. WHO. International statistical classification of diseases and related health problems ICD-10: instruction manual. Vol. 2. 2016.

12. Karnofsky DA, Abelmann WH, Craver LF, Burchenal JH. The use of the nitrogen mustards in the palliative treatment of carcinoma. With particular reference to bronchogenic carcinoma. Cancer. 1948;1(4):634-56.

13. Conill C, Verger E, Salamero M. Performance status assessment in cancer patients. Cancer. 1990;65(8):1864-6.

14. Cure GC, Aristizabal G, Aristizabal J, Roa CL, Alvarado H. Características demográficas y patológicas de los tumores del sistema nervioso central estudiados en la clínica El Bosque. Acta neurol colomb. 2011;27(2):106-13.

15. da Cunha MLV, Grosbelli L. Profile of Patients with Intracranial Tumors Undergoing Surgical Resection at a Neuro-oncology Referral Hospital. Arq Bras Neurocir. 2018;37(01):19-26.

16. Ramos-Clason EC, Tuñón-Pitalua MC, Rivas-Muñoz FA, Veloza-Cabrera LA. Tumores primarios del sistema nervioso central en Cartagena, 2001-2006. Rev Salud Pública. 2010;12:257-67.

17. Pardo C, Cendales R. Incidencia, mortalidad y prevalencia de Cáncer en Colombia, 2007-2011. Primera ed. Bogotá. D.C.: Instituto Nacional de Cancerología; 2015. 148 p.

18. Piñeros M, Sierra MS, Izarzugaza MI, Forman D. Descriptive epidemiology of brain and central nervous system cancers in Central and South America. Cancer Epidemiol. 2016;44:S141-9.

19. Newton HB, Otero JJ. Overview of Epidemiology, Pathology, and Treatment of Primary Brain Tumors. In: Newton HB, Maschio M, editor. Epilepsy and Brain Tumors [Internet]. Boston: Academic Press; 2015. p. 11-28. Available from: http://www. sciencedirect.com/science/article/pii/B978012417043800002X 
20. Barnholtz-Sloan JS, Ostrom QT, Cote D. Epidemiology of brain tumors. Neurol Clin. 2018 Aug;36(3):395-419.

21. Westphal M, Lamszus K, Tonn JC. Meningiomas and meningeal tumors. In: Tonn JC., Westphal M., Rutka JT, editor. Oncology of CNS tumors. Berlin, Heidelberg: Springer; 2010. p. 95-118.

22. Hatef J, Adamson C, Obiga O, Taremwa B, Ssenyojo H, Muhumuza M, et al. Central nervous system tumor distribution at a tertiary referral center in Uganda. World Neurosurg. 2014;82(3-4):258-65.

23. Qi Z-Y, Shao C, Huang Y-L, Hui G-Z, Zhou Y-X, Wang Z. Reproductive and exogenous hormone factors in relation to risk of meningioma in women: a meta-analysis. PLoS One. 2013:8(12):e83261.

24. Maclean J, Fersht N, Singhera M, Mulholland P, McKee O, Kitchen N, et al. Multi-disciplinary management for patients with oligometastases to the brain: results of a 5 year cohort study. Radiat Oncol. 2013:8(156):1-8.

25. Fabi A, Felici A, Metro G, Mirri A, Bria E, Telera S, et al. Brain metastases from solid tumors: disease outcome according to type of treatment and therapeutic resources of the treating center. J Exp Clin Cancer Res. 2011;30(1):1-7.

26. Fernández de Aspe P, Fernández Quinto A, Guerro Moya A, Arán Echabe E, Varela Pazos A, Peleteiro Higuero $P$, et al. Experiencia en el manejo de metástasis cerebrales tratadas con radiocirugía estereotáctica TT. Neurocirugia. 2016;28(2):75-87.

27. Rodrigues D, de Oliveira Lima L, Pereira E, de Oliveira Souza U, de Oliveira M, Fernandes de Moura Lima A, et al. Epidemiologia das neoplasias intracranianas no Hospital do Servidor Público Estadual de São Paulo: 2010-2012. Arq Bras Neurocir. 2014;33(01):6-12.

28. Fabi A, Vidiri A, Ferretti G, Felici A, Papaldo P, Carlini P, et al. Dramatic Regression of Multiple Brain Metastases from Breast Cancer with Capecitabine: Another Arrow at the Bow? Cancer Invest. 2006;24(4):466-8.

29. Páez A, Erazo-Bravo N, Merchancano-Delgado C. Caracterización de los tumores cerebrales en un Hospital Universitario de Pereira, Colombia: un estudio retrospectivo. Rev Médica Risaralda. 2013;19(2):8. 